\title{
Porous poly(lactic acid) based fibres as drug carriers in active dressings
}

\author{
EWA DZIERZKOWSKA ${ }^{1}$, ANNA ŚCISŁOWSKA-CZARNECKA ${ }^{2}$, SARA MATWALLY ${ }^{3}$, \\ DOROTA ROMANISZYN ${ }^{4}$, MAGDALENA CHADZIŃSKA ${ }^{5}$, EWA STODOLAK-ZYCH ${ }^{1} *$ \\ ${ }^{1}$ AGH University of Science and Technology, Faculty of Materials Science and Ceramics, Kraków, Poland. \\ ${ }^{2}$ Academy of Physical Education, Department of Physiotherapy, Section of Cosmetology, Kraków, Poland. \\ ${ }^{3}$ International Centre of Electron Microscopy for Material Science, AGH University of Science and Technology, \\ Faculty of Metals Engineering and Industrial Computer Science, Kraków, Poland. \\ ${ }^{4}$ Jagiellonian University Medical College, Department of Microbiology, Kraków, Poland. \\ ${ }^{5}$ Jagiellonian University, Faculty of Biology, Institute of Zoology and Biomedical Research, Kraków, Poland.
}

\begin{abstract}
Purpose: The polymeric porous surface of fibres (PLA) may influence the kinetics of release of biologically active compounds (gentamicin, G and ethacridine lactate, R) affecting development of a bacterial biofilm. Methods: The porous fibres with different morphology were manufactured by the electrospinning method from ternary systems composed of PLA and selected solvents. Fibres morphology was examined using a scanning electron microscopy (SEM), their structure was analyzed by FT-IR ATR spectroscopy and differential scanning calorimetry (DSC). Changes in the drug release profile were measured using ICP/UV-Vis methods and the resulting bactericidal or bacteriostatic properties were tested by two-layer disk diffusion test in relation to various drug incorporation methods. Results: The porous fibres can be applied to produce drug-bearing membranes. The spectroscopic studies confirmed incorporation of gentamicin into the fibres and the presence of ethacridine lactate on their surface. Bimodal fibres distribution (P3) promoted faster release of gentamicin and ethacridine lactate from P3G and P3R materials. The electrospinning process coupled with the vapor induced phase separation influenced the glass transition temperature of the porous polymer fibres. The pre/post-electrospinning modification influenced the glass transition, maximum temperature of cold crystallization and melting point of the porous membrane, compared to the neat polymer. The polylactide fibres with gentamicin showed strong bactericidal effect on Gram-positive bacteria, while fibres with ethacridine lactate were bacteriostatic. Conclusions: The obtained fibres with complex surface morphology can be used as a membrane in active dressings as they make it possible to control the release profile of the active compounds.
\end{abstract}

Key words: wound dressing, active dressing, nanostructured fibres, electrospun polylactide, drug delivery

\section{Introduction}

A chronic wound, unlike an acute wound, is a long-healing wound whose prolonged healing process causes bacteria adhesion and biofilm formation [10], [25]. The first pathogens that attack wounds are Grampositive bacteria, coming from the surrounding area or directly from the skin, and then, in the next stage, in addition to Gram-positive bacteria, Gram-negative bacteria appear on the wound, initiating a complex inflammatory process and, as a consequence, tissue destruction. It is predicted that in 2050 more deaths will occur as a result of bacterial infections than as a result of cancer [24]. These predictions have contributed to the development of the concept of a wound dressing that combines two features, i.e., reconstruction of the native ECM (extracellular matrix) microstructure and the creation of a physical barrier containing antimicrobial agent against bacterial colonization. On one hand, fibrous scaffold can be a structure that mimics the extracellular matrix (ECM), on the other

* Corresponding author: Ewa Stodolak-Zych, AGH University of Science and Technology, Faculty of Materials Science and Ceramics, ul. A. Mickiewicza 30, 30-059 Kraków, Poland. Phone: +48 12 6173441, fax: +48 1261733 71, e-mail address: stodolak@agh.edu.pl

Received: January 22nd, 2020

Accepted for publication: May 11th, 2020 
hand, fibres can be carriers of active compounds that inhibit biofilm formation and promote wound healing.

New concept of fibrous structure used in biomedical application are nanofibers which are fibres with a diameter in the range of 50-1000 nm, several orders of magnitude smaller than fibres produced by conventional spinning methods [1], [14]. Nanofibres have become increasingly attractive, mainly due to their very porous structure and large surface-to-volume ratio [15], [17], [26]. Membranes made of electrospun fibres are characterised by high roughness resulting from the random distribution of fibres, but this surface development is not sufficient to prevent biofilm formation. However, careful selection of solvents enables achieving porous fibres, and the method based on the ternary system of nonsolvent/solvent/polymer and high humidity during the electrospinning process has been precisely described elsewhere [11], [14], [18]. A number of benefits can be derived from this microstructure, such as: reduction of bacterial adhesion, as well as a modified drug release profile compared to solid fibres [21], [26].

Nanofibres are fabricated in an electrospinning process, based on the ejection of a polymer solution jet due to the high electrical field applied between the needle and the collector. Nonwoven membranes are characterized by a high surface area to volume ratio, high permeability and tailorable fibre diameters [1], [17]. Hence, four types of wound dressing based on electrospun fibres are classified as follows: passive dressing, interactive dressing, advance dressing and smart dressing. The most popular passive dressing produced from natural or synthetic polymers provides only the suitable environment due to its porous microstructure and protection against the external environment. By modifying synthetic polymer fibres with biological molecules, an interactive dressing can be obtained. The advanced dressing contains fibres and a drug that can inhibit bacteria adhesion and colonization.

The modification of fibres with active component agents is an option for inhibiting bacterial infection. The division is based on pre-electrospinning and postelectrospinning modification [17]. The pre-electrospinning involves encapsulation of active agents dissolved or dispersed in the polymer solution. The postelectrospinning method involves chemical immobilization, physical adsorption or layer-by-layer assembly [11]. Both methods of modification of electrospun fibres can be obtained with polylactide. Moreover, the use of this polymer ensures good electrospinnability, and the form of fibrous membrane is favourable for wound healing. Sustained drug delivery from this polymer was widely reported [11], [18], [28]. Polylac- tide has been used for years in biomedical engineering as a material that in the form of screws, plates, scaffoldings or implant coatings finds clinical application and has the appropriate approvals (e.g., FDA test) [6], [25]. Due to thermoplastic properties, the polymer can be formed by methods typical for polymer processing (injection, extrusion, 3D printing) as well as methods that use its good solubility in organic solvents [10], [16], [24]. The latter option makes it possible to change the surface morphology of the fibre. There is another "promising" possibility of using nanostructured materials. Bacterial interaction with a highly developed, rough surface is inverse to interaction with smooth surfaces [3], [7], which is influenced by adhesion energies, adhesion forces, and associated cell wall deformation. In addition, other polyhydroxy acids, e.g., PGA or PLGA, despite high safety (FDA approval for the introduction of polymers into clinical practice) [13], [24] do not create so many ternary systems that allow for implementation of electrospinning combined with the phase inversion method (NIPS, VIPS) [13], [18], [29]. Disadvantage of the neat PLA is weak antimicrobial activity [15].

The increasing resistance of bacteria to antibiotics means that their topical application should take place only in justified cases - when there are no other methods to combat the bacteria. In addition, topical antibiotics usually act against a small group of bacteria and have a difficult penetration of the biofilm, hence antiseptics (e.g., ethacridine lactate, octenidine, polyhexanidine and iodine compounds) are much more effective in the first contact with the wound [9]. Their task is to protect the wound by killing microbes or/and inhibiting their growth, which affects the activation of processes associated with the regeneration of damaged tissue. Antiseptics do not affect the emergence of resistance in bacteria (i.e., as a result of the action of antiseptics, bacteria resistant to commonly available antibiotics do not appear), but, due to the short duration of activity their effectiveness is limited (max several hours) [12], [20].

In our research, we used two active substances: an antibiotic (gentamicin sulphate, G) and an antiseptic (ethacridine lactate, R). Research using these substances was carried out due to the planned combining of the dressing layers at a later stage: the first would be antiseptic (PLA with ethacridine lactate) while the second should be bactericidal (PLA with gentamicin).

Commercially available $0.1 \%$ ethidium lactate solution (Rivanol, Fig. 1b) is antiseptic fluid that acts on bacterial DNA (binds to it, making bacterial division difficult, bacteriostatic). It is particularly effective against Gram-positive bacteria and, what is im- 
portant, it works even in the environment of organic substances such as exudate, blood and serum [8], [19]. Ethidium lactate is an aseptic that has been widely used for years and causes no controversy. All remarks in the literature related to the method of application to the wound (carrier: cotton wool/gauze/ hydrogel, e.g., PVP) and the colour effects of dressings with ethacridine lactate [12], [19]. Unfortunately, its bactericidal properties are good as soon as the solution is prepared and worsen with time.

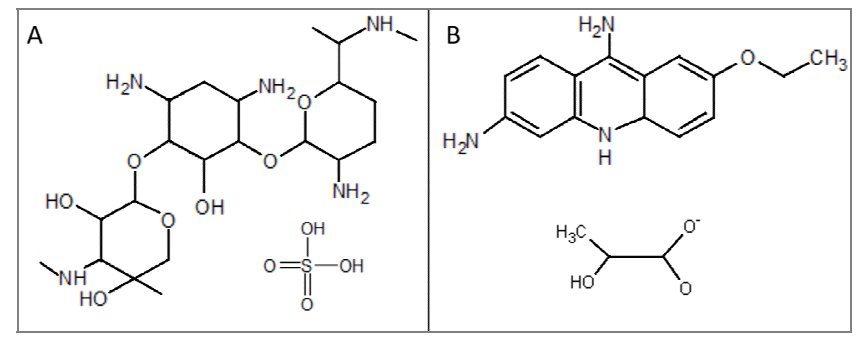

Fig. 1. Structural formula: gentamicin sulphate (A) and ethactin lactate (B)

The second active agent: gentamicin sulphate (Fig. 1a) is used in the form of a solution for skin wounds [4], [22]. In dermatology, gentamicin is used to treat skin inflammations with exudate complicated by secondary bacterial infection. Due to the fact that the formation of resistant bacterial strains is much greater than after parenteral administration, gentamicin is recommended as a last resort. Direct application of topical antibiotic solution is much more effective than the administration in the form of an ointment or gel [2], [4]. After a few days of use, epithelial epithelialization is observed, and the wound remains clean and free from bacterial biofilm of Staphylococcus aureus and Pseudomonas aeruginosa [22]. Notably, topical antibiotic not only does not affect renal function, but minimizes the risk of developing resistance pathogens, and has the ability to kill bacteria by inhibiting protein synthesis [19], [21], [25].

The aim of this study was to obtain two kinds of porous fibres with different porosity, which were modified by gentamicine $(\mathrm{G})$ and ethacridine lactate (R). Gentamicin used in the study is not soluble in any of the solvents used in the experiment. Gentamicin particles were homogenised in an electrospinning polymer solution prior to the electrospinning process (pre-electrospinning modification). Ethacridine lactate is a liquid, so membranes consisting of porous and nano-porous fibres have been modified by surface soaking (post-electrospinning modification). Nanostructured fibres and various drug incorporation methods have changed the drug release profile and influenced the resulting bactericidal or bacteriostatic properties.

\section{Materials and methods}

Polylactide 3251D (PLA) Mw of $55.4 \mathrm{~kg} / \mathrm{mol}$ and isomer $\mathrm{D}$ lactic acid content of $1.2 \%$ purchased from Nature Works were used in the study. This commercial PLA has glass transition temperature of $55-60{ }^{\circ} \mathrm{C}$. The polymer with three sets of solvents, i.e., dichloromethane (DCM, high purity), dimethylformamide (DMF, high purity), chloroform (CHL, high purity) and dimethyl sulfoxide (DMSO, high purity) supplied by Avantor SA as base solutions for the electrospinning process. Polymer concentrations and solvents ratios have been studied in earlier works [27], [28]. The electrospinning process was carried out on an EC-DIG electrospinning apparatus with a climate chamber system (IME Technologies, The Netherlands) at $25{ }^{\circ} \mathrm{C}$ and $30 \%$ relative humidity for $\mathrm{P} 1 \mathrm{ma}-$ terial and $70 \%$ for other materials (P2 and P3). For all materials used, the applied voltage was in the range of $11-15 \mathrm{kV}$, the flow of polymer solution from the syringe pump was $1 \mathrm{ml} / \mathrm{h}$, and the distance between the metal nozzle (inner diameter of $0.8 \mathrm{~mm}$ ) and the rotating drum collector was $17 \mathrm{~cm}$.

In the pre-electrospinning modification, the appropriate PLA solutions were sonicated with $5 \mathrm{wt} . / \mathrm{v} \%$ of gentamicin (Polfa S.A., Poland), and thus prepared solutions were used to obtain fibres named: P1G, P2G and P3G. In the post-electrospinning modification, both porous (P2, P3) and non-porous fibres (P1) were modified by soaking into a solution of ethacridine lactate (Rivanol 0.1 wt./v \%, HASCO-LEK S.A., Poland) for $24 \mathrm{~h}$ at $20^{\circ} \mathrm{C}$ to obtain fibres named: P1R, P2R and P3R. All materials used in the study were membranes with multidirectional fibre arrangement, characterized by a porous or non-porous fibre surface (Table 1).

Prior to SEM investigation, the samples were coated with a layer of gold approximately $5 \mathrm{~nm}$ thick, using a rotary-pumped sputter coating (Q150RS, Quorum Technologies, UK). The morphology of modified PLA fibres was evaluated by SEM (Merlin Gemini II, Zeiss, Germany) applying a voltage of $3 \mathrm{kV}$ and current of $20 \mathrm{pA}$. Fibres diameters were measured based on SEM images using Fiji Lifeline (ver. 2015 December 22, USA). The mean diameter was calculated based on 100 measurements with an error based on standard deviation. The presence of bacteria on the 
Table 1. Composition of polymer solutions for electrospinning

\begin{tabular}{|l|c|c|c|}
\hline \multicolumn{1}{|c|}{ Type of membrane } & Material & Solvents ratio & $\begin{array}{c}\text { Fibre } \\
\text { microstructure }\end{array}$ \\
\hline \multirow{3}{*}{ Neat polymer fibrous membrane (reference) } & P1 & DCM:DMF, 7:3 & non-porous fibres \\
\cline { 2 - 4 } & P2 & CHL:DMSO, 4:1 & porous fibres \\
\cline { 2 - 4 } & P3 & CHL:DCM, 3:1 & porous fibres \\
\hline \multirow{2}{*}{$\begin{array}{l}\text { Polymer fibre modified in volume with } \\
\text { gentamicine }(G)\end{array}$} & P1G & DCM:DMF, 7:3 & non-porous fibres \\
\cline { 2 - 4 } & P2G & CHL:DMSO, 4:1 & porous fibres \\
\cline { 2 - 4 } & P3G & CHL:DCM, 3:1 & porous fibres \\
\hline \multirow{2}{*}{$\begin{array}{l}\text { Polymer fibre modified on the surface with } \\
\text { ethacridine lactate }(R)\end{array}$} & P1R & DCM:DMF, 7:3 & non-porous fibres \\
\cline { 2 - 4 } & P2R & CHL:DMSO, 4:1 & porous fibres \\
\cline { 2 - 4 } & P3R & CHL:DCM, 3:1 & porous fibres \\
\hline
\end{tabular}

fibres after the bacteriological tests was checked using Nova Nano SEM 200 (FEI Company) with graphite coating.

The structural characteristics of the membranes were performed by Fourier transformation infrared spectroscopy with an attenuated total reflectance accessory (FTIR-ATR, FT 3000 Excalibur). The test was carried out on a diamond crystal in the wavenumber range of $500-4000 \mathrm{~cm}^{-1}$ and resolution of $4 \mathrm{~cm}^{-1}$.

Differential scanning calorimetry of the fibrous membrane were performed using DSC1 calorimeter from Mettler Toledo on samples of ca. $3.5 \mathrm{mg}$ placed on aluminium sample pans. The measurements were performed in the temperature range from 0 to $210^{\circ} \mathrm{C}$, with heating and cooling rates $10^{\circ} \mathrm{C} / \mathrm{min}$. The under a dynamic nitrogen atmosphere (flow rate of $30 \mathrm{ml} / \mathrm{min}$ ).

Drug release experiments were performed by inductively coupled plasma spectroscopy (ICP-ASA, ICP 4500, Hewlett-Packard) for gentamicin-modified fibrous membrane. Sulphate ions (derived from gentamicin sulphate) were used as analytical ions and their concentration after 12 weeks of incubation in PBS (phosphate buffer solution, Merck) was measured based on 5 measurements in an ICP spectrometer and the standard deviation was calculated.

Drug release experiments were performed using UV-Vis spectrophotometry (CE 2325, Cecil Instr.) for membranes with ethacridine lactate. Small pieces of the membranes, each $2.5 \times 1.5 \mathrm{~cm}$ in size, were incubated for 12 weeks at $37{ }^{\circ} \mathrm{C}$ in $30 \mathrm{ml}$ of phosphate buffer saline medium (PBS, MERCK) in polypropylene tubes. The calibration curves for the UV-Vis examination were established by measuring the absorbance of ethacridine lactate solutions (reference solutions) at different concentrations for wavelength $368 \mathrm{~nm}$. The concentration of ethacridine lactate in the samples after incubation was obtained based on calibration curves.
The burst release of the drug (gentamicin) was observed during short term observations conducted for 7 days $\left(37^{\circ} \mathrm{C}\right.$ in $30 \mathrm{ml}$ of phosphate buffer saline medium, PBS), taking a daily dose of the medium and subjecting it to measurement in an ICP spectrometer. The time of monitoring the release of ethacridine lactate to the incubation medium (PBS) was $8 \mathrm{~h}$ (time of maximum action of the antibacterial agent on the wound). The change of ethacridine lactate concentration was determined on the basis of UV-Vis measurements.

The purpose of the two-layer disk diffusion test was to determine the antimicrobial activity of membranes modified with drugs. Disks having diameter of $6 \mathrm{~mm}$ of all membranes were tested against Escherichia coli ATCC 25922 (Gram-negative bacteria) and Staphylococcus aureus ATCC 29213 (Gram-positive bacteria). Test bacteria were cultured on Trypticasein Soy LAB-AGAR (TSA, Biocorp) on a Petri dish at $35 \pm 2{ }^{\circ} \mathrm{C}$ in an atmosphere of $5 \% \mathrm{CO}_{2}$ for $24 \mathrm{~h}$. Two or three colonies were diluted in $0.9 \% \mathrm{NaCl}$ to obtain 2.3 in McFarland measured using densitometers for the measurements of turns of cells suspension (DEN1, BioSan). In the first step, plates having a diameter of $6 \mathrm{~mm}$ were prepared with a first layer of $10 \mathrm{ml}$ of pure TSA agar and allowed to dry. A second layer of $10 \mathrm{ml}$ of inoculated TSA agar with prepared suspension (150 $\mathrm{ml}$ of TSA with $1 \mathrm{ml}$ of bacterial suspension) was applied onto the first one. Fibrous membrane disks were placed on dry inoculated agar plates and then incubated for $24 \mathrm{~h}$ at $35 \pm 2{ }^{\circ} \mathrm{C}$ in $5 \% \mathrm{CO}_{2}$, after which the diameters of the inhibition zones were measured. Prior to SEM testing, samples after incubation were removed from agar, rinsed with PBS and fixed with $2.5 \%$ glutaraldehyde solution (Sigma Aldrich) for $2 \mathrm{~h}$. Then the samples were dehydrated in water-alcohol solutions with a gradually increasing ethanol concentration $(50 \%, 70 \%, 96 \%)$ three times for each concertation for $3 \mathrm{~min}$. 


\section{Results}

Based on the microstructural analysis of gentamicin sulphate, it can be stated that the aminoglycoside antibiotic was characterized by a spherical morphology, with a particles size in the range of $10-100 \mathrm{~nm}$ (Fig. 2). Gentamicin sulphate is soluble in water $(50 \mathrm{mg} / \mathrm{ml})$, and is neither soluble in DMSO, DCM, DMF nor in CHL, which means that gentamicin can be treated as a particle introduced into the polymeric matrix, which in this particular case is fibrous. Ethacridine lactate, which has lower solubility in water $(0.192 \mathrm{mg} / \mathrm{ml})$ than gentamicin and tends to secondary agglomeration in both polar and non-polar solvents [2], [22]. For this reason, ethacridine lactate was used in the present study as a commercial solution in the post - electrospinning modification of fibres (fibrous membrane).

In the electrospinning process, from the ternary system of nonsolvent/solvent/polylactic acid, porous and non-porous fibres with three significantly different microstructures were obtained. From neat polymer, and their morphology was presented in Fig. 3. The fibre arrangement in all electrospun membranes was random and the fibre diameters, surface porosity and roughness depended on the solvent system. The P1 membrane based on the PLA:DCM:DMF system had solid fibres with a diameter of approx. $0.84 \mu \mathrm{m}$ and a slightly rough surface. The fibres obtained from the PLA:CHL:DMSO system (named P2) had an uniform diameter of $2 \mu \mathrm{m}$, and the use of this ternary system made it possible to obtain highly porous fibres with a pore diameter of approx. $126 \mathrm{~nm}$ (Fig. 4). The most heterogeneous P3 materials were characterized by two modes of fibres diameters and were obtained from the PLA:CHL:DCM system. The P3 fibres can be divided into two populations, i.e., the first consisting of smaller fibres with a diameter up to $1,5 \mu \mathrm{m}$ and the second with larger fibres having a diameter above $6 \mu \mathrm{m}$.

The presence of porous fibres allowed the introduction of ethacridine lactate into the fibres surface during post-electrospinning modification (soaking). The incorporation of ethacridine lactate did not affect the morphology of fibres therefore SEM microphotographs of such material were not presented.

The addition of gentamicin caused a slight increase in the diameter of fibres from 0.84 (for P1) to $0.89 \mu \mathrm{m}$ (for $\mathrm{P} 1 \mathrm{G}$ ) and did not affect the surface roughness (Fig. 4). In the case of porous fibres named P2G, a slight decrease in the diameter of the fibres and an increase in the pore diameters were observed compared to the same fibres without the addition of the filler (gentamicine). Furthermore, the addition of the gentamicin increased both the diameter of the fibres and the pores.

Significant differences in fibre morphology and size distribution are visible for the P3 type fibres when modified with gentamicin. The addition of active filler (gentamicin) does not affect the size distribution, which is still bimodal. The average fibre diameter increases from about $1.5 \mu \mathrm{m}$ (visible in P3) to $1.65 \mu \mathrm{m}$ (for $\mathrm{P} 3 \mathrm{G}$ ). The second fibre population maintains a similar size (diameter of about $6.2 \mu \mathrm{m}$ ), increasing from $10 \%$ (for $\mathrm{P} 1$ ) to $23 \%$ (for $\mathrm{P} 3 \mathrm{G}$ ), which may also indicate that gentamicin agglomerates during the electrospinning process. Hence, the larger
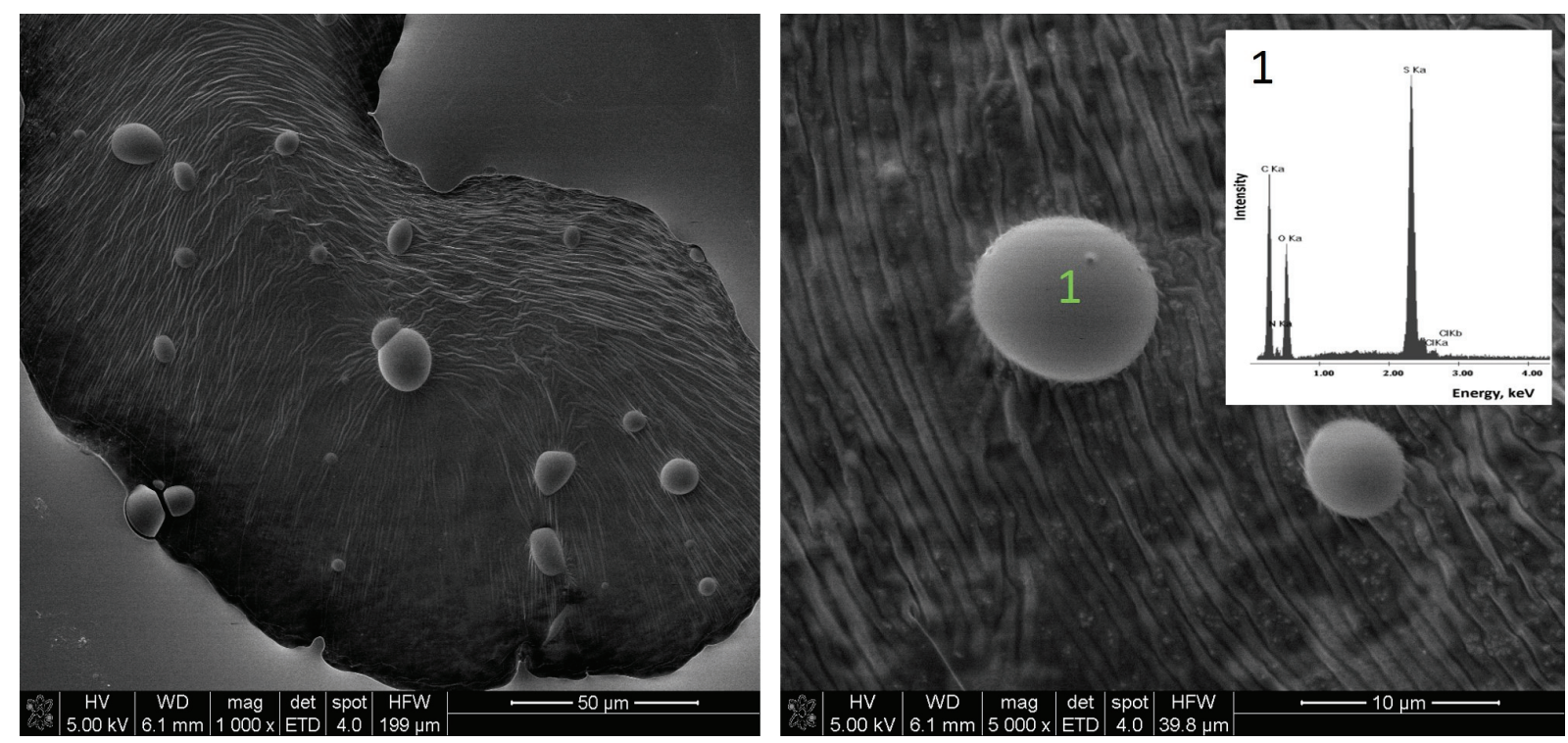

Fig. 2. Morphology of gentamicine sulphate and their EDS spectrum 

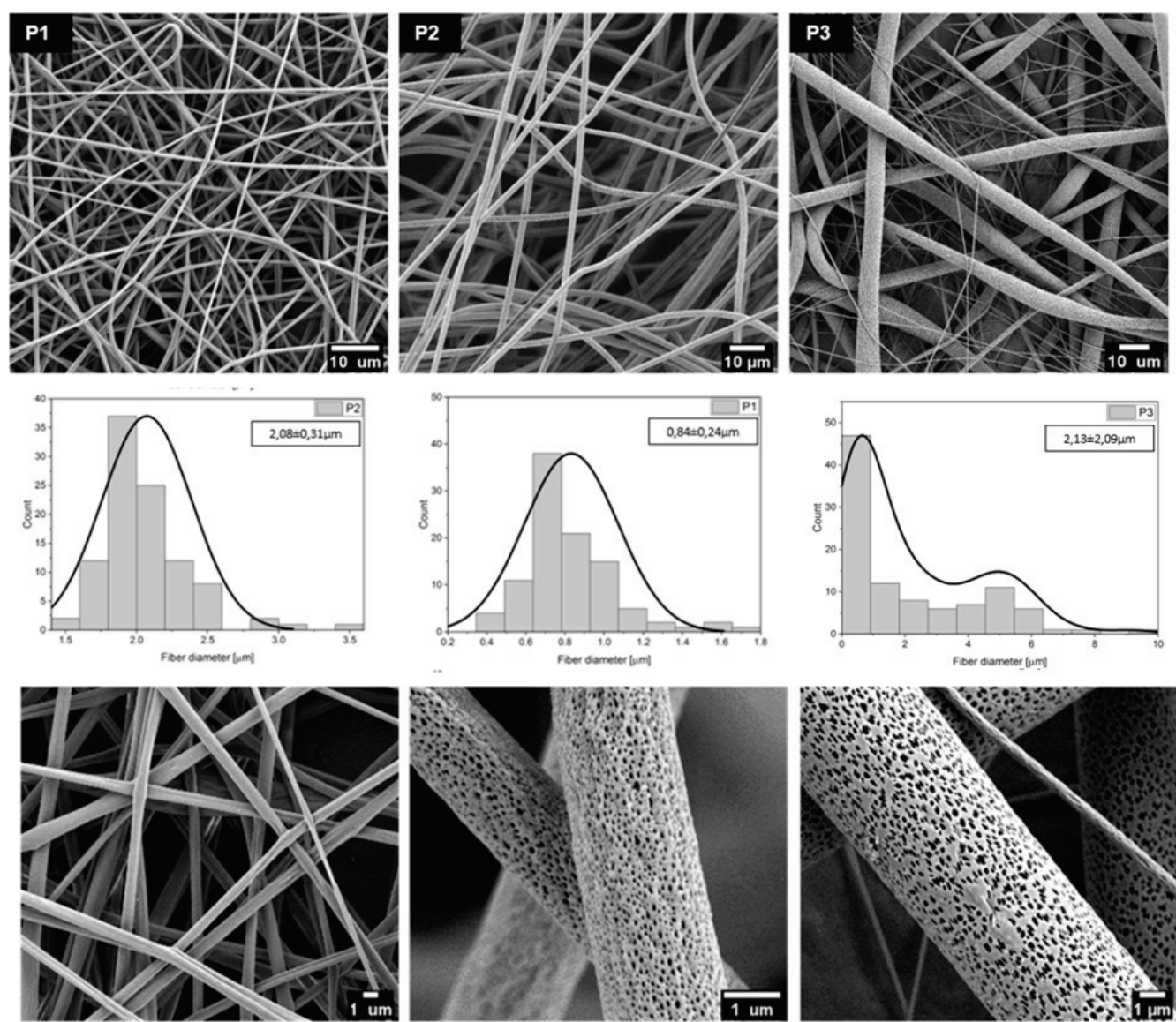

Fig. 3. Microphotographs of pure PLA fibres: non-porous (P1) and porous (P2, P3) with fiber size distribution
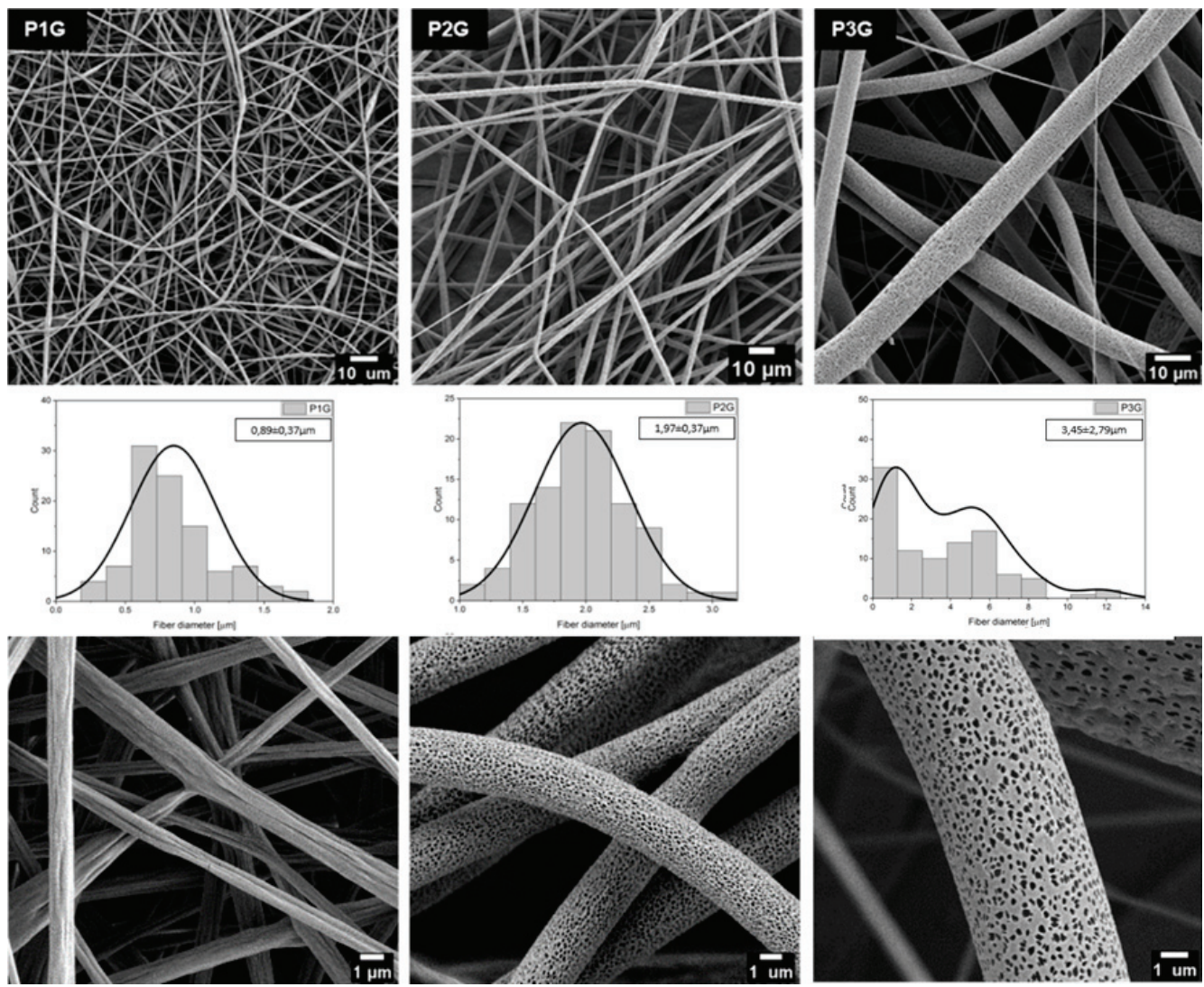

Fig. 4. Microphotographs of; PLA fibres modified by gentamicin: non-porous (P1G) and porous (P2G, PG3) with fibres size distribution 

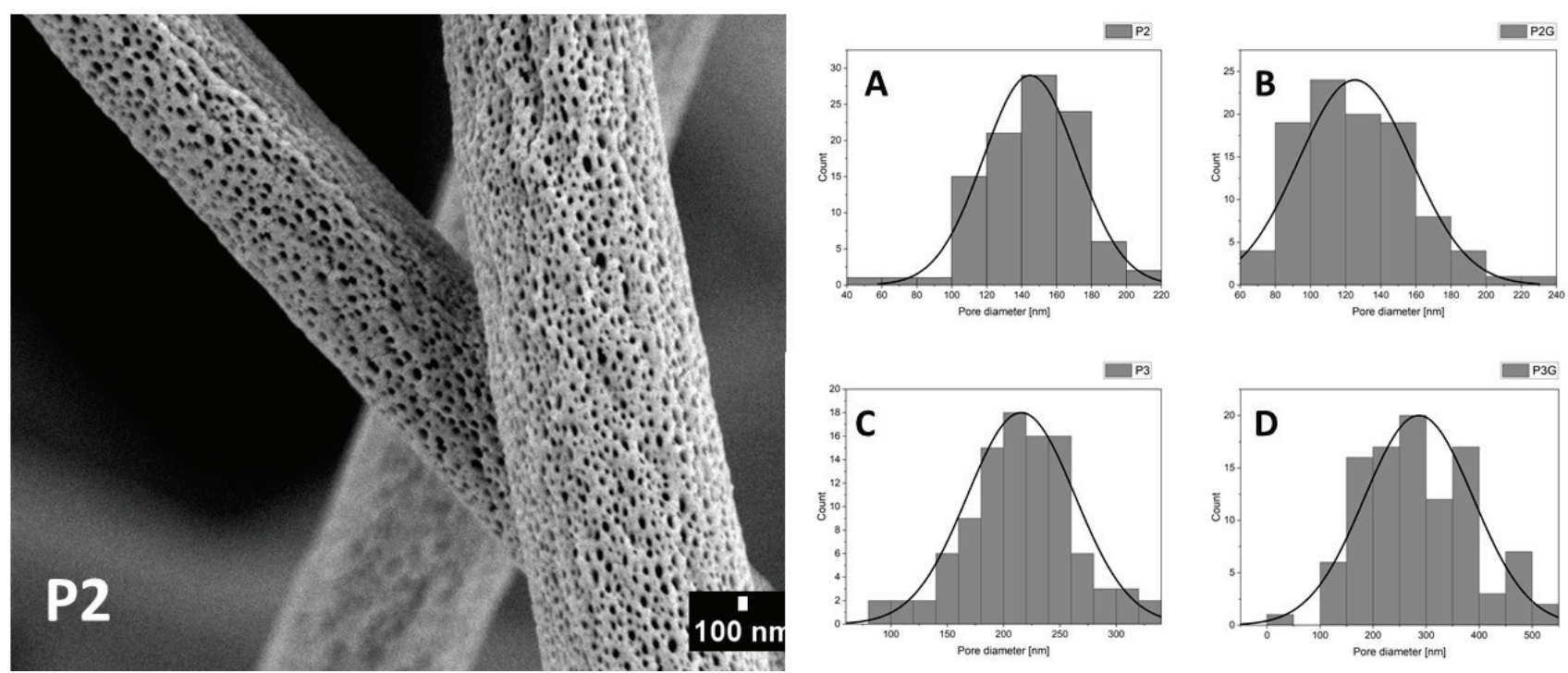

Fig. 5. Microstructure of PLA fibres (P2) with pore size distribution in fibres unmodified (P2 -A, P3-C) and modified with gentamicine (P2G-B and P3G-D)
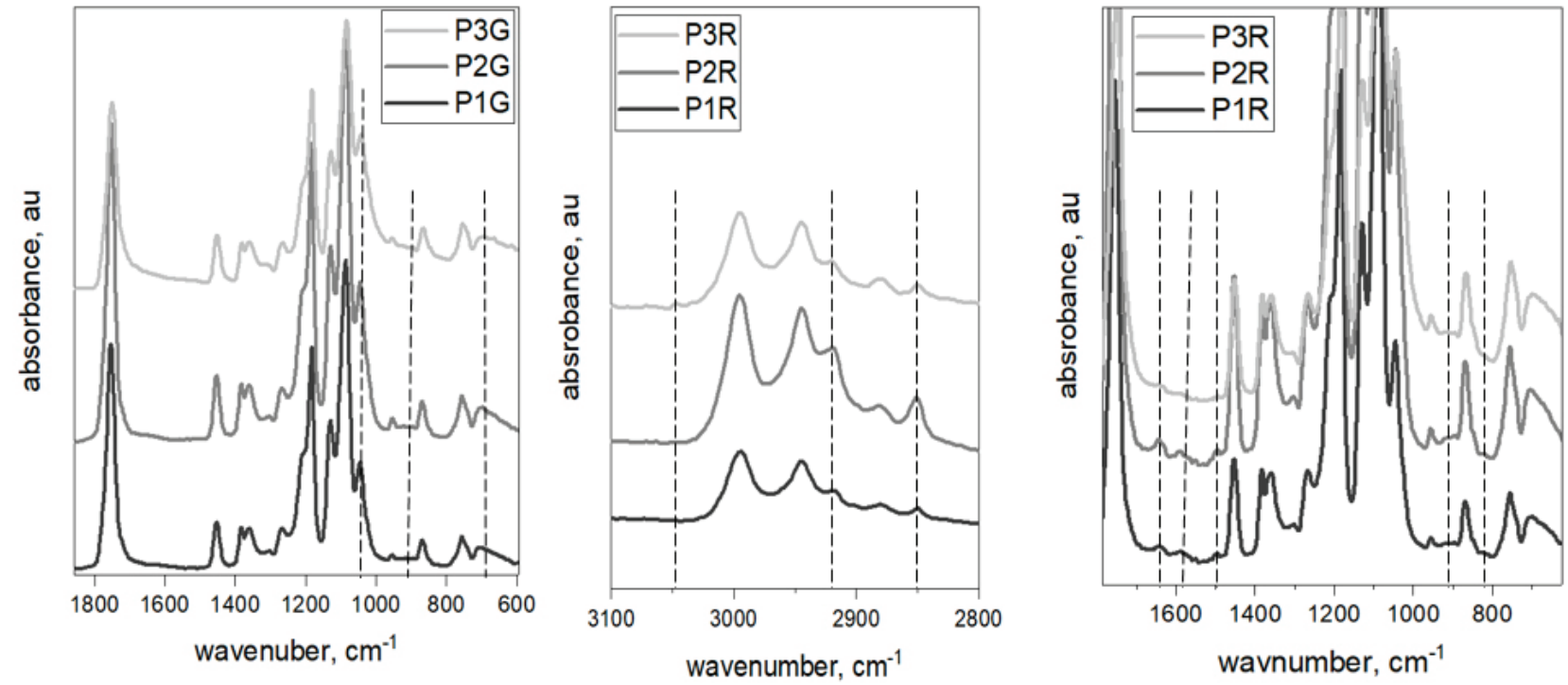

Fig. 6. FTIR spectra of PLA fibre with: a) gentamicin (region $1800-600 \mathrm{~cm}^{-1}$ ),

b) ethacridine lactate (region $3100-2800 \mathrm{~cm}^{-1}$ ), c) ethacridine lactate (region 1800-600 $\mathrm{cm}^{-1}$ )

fibre diameter influenced the pore size, and the P3 fibres had larger pores compared to the P2 fibres. Pore sizes and fibre porosity estimated based on SEM images were presented in Figs. 5a-d.

The $\mathrm{P} 2 \mathrm{G}$ fibres were characterized by a high porosity $(\sim 42 \%)$ and the monomodal pore size distribution with the modal pore diameter of approx. 126 nm (Fig. 5B), which was like the pore size distribution in the P2 fibres (Fig. 5A). Pores in the P3G fibres were larger (267 nm, Fig. 5C) than in and the P3 fibres (Fig. 5D), but their porosity was similar i.e., $\sim 35 \%$.

FTIR-ATR spectroscopic study was performed to confirm the successful incorporation of gentamicin into the fibres and the presence of ethacridine lactate on their surface are presented in Fig. 6. The FTIR-ATR spectra of the modified PLA membrane in which the characteristic vibrations representing gentamicin or ethacridine lactate are visible.

Differential scanning calorimetry was performed on all materials to confirm the presence of drugs. The influence of drugs on characteristic temperatures ( $T_{g}$ - glass transition temperature, $T_{c}-$ temperature of cold crystallization, $T_{m}$ - melting point temperature) is summarized in Table 2. The degree of crystallinity $\left(X_{c}\right)$ was determined on the basis of the melting enthalpy of fully crystalline polylactide, which is $93 \mathrm{~J} / \mathrm{g}$ [23]. 

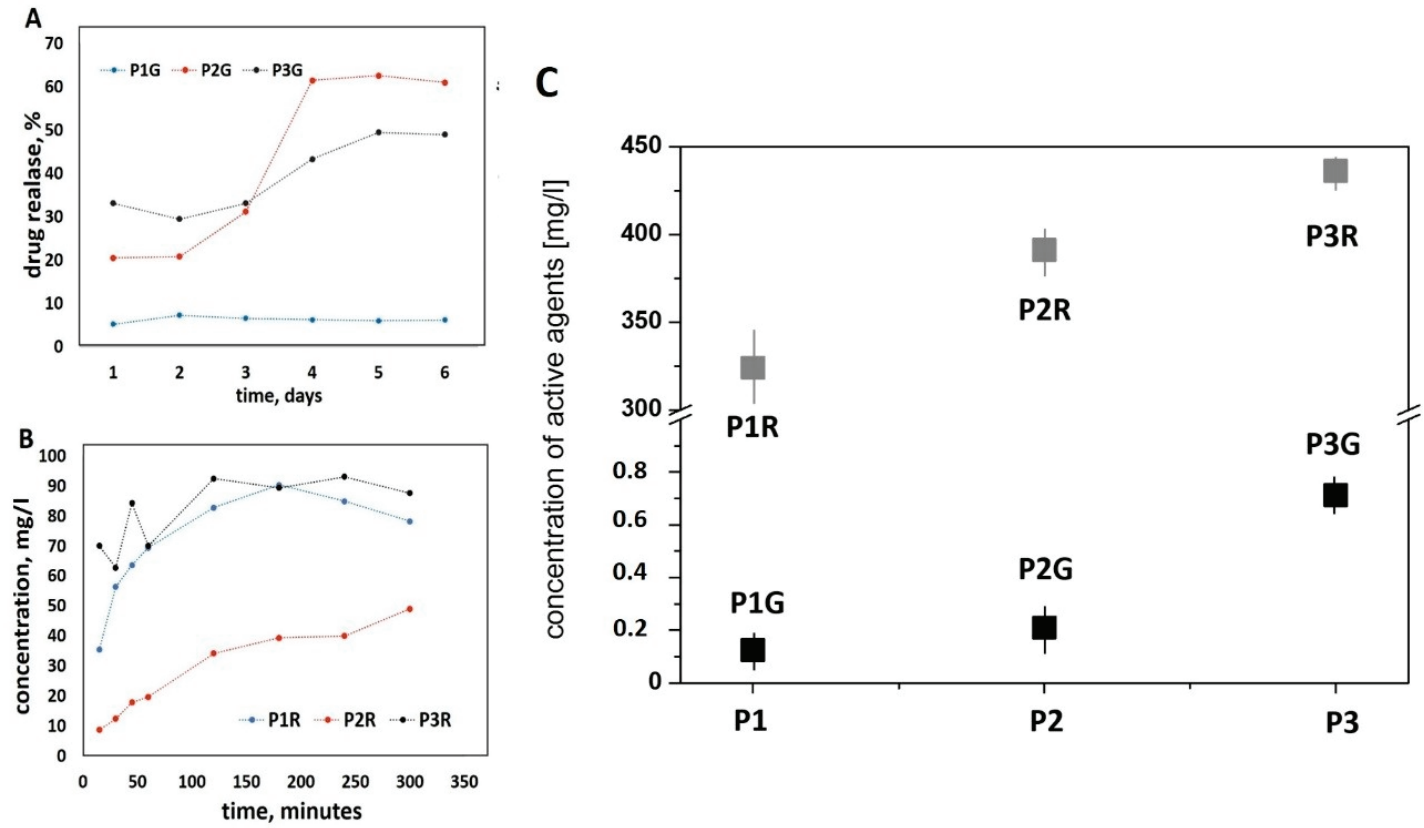

Fig. 7. Release of the active agents: gentamicin and ethacridine lactate from fibrous membranes made of non-porous and porous fibres: A - cumulative drug release of gentamicine during 7 days, B - concentration of antiseptic agents for 5 hours,

$\mathrm{C}$ - changing of concentration of active agents during the 12 weeks studied.

Note: The graph shows the concentration of active agents in $\mathrm{mg} / \mathrm{l}$ due to significant differences in the amount of active agent released, the concentration axis has two scales: the first region $-0-1.0 \mathrm{mg} / \mathrm{l}$ for gentamicin (explicitly a sulfonic ion), the second region: $300-450 \mathrm{mg} / \mathrm{l}$ for ethacridine lactate (explicitly the colour of ethacridine lactate)

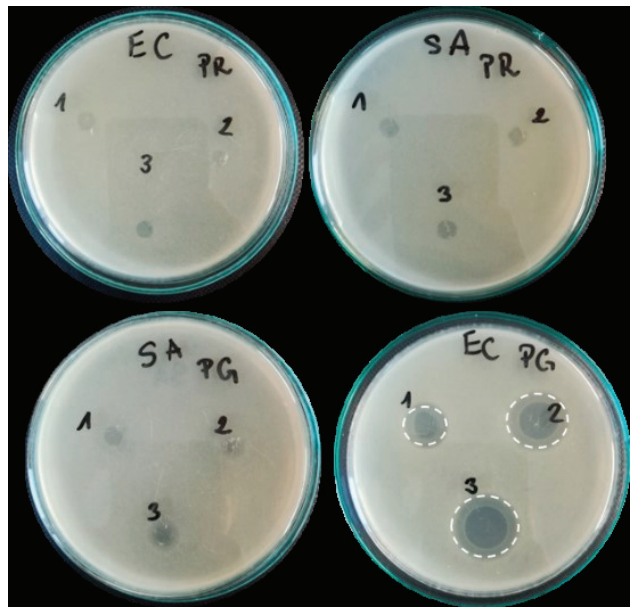

Fig. 8. Zones of inhibition of bacteria growth around fibrous membranes modified with gentamicin (P1G, P2G, P3G) and ethacridine lactate (P1R, P2R, P3R) against E. coli and $S$. aureus bacteria

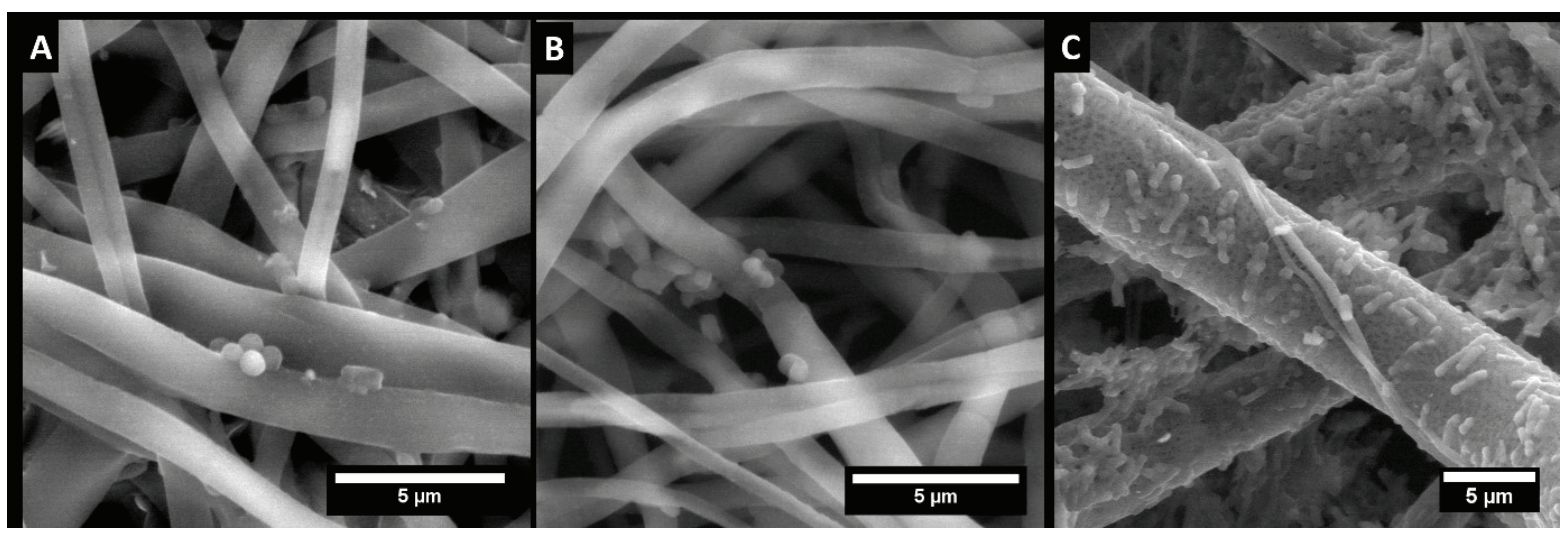

Fig. 9. SEM microphotographs of fibrous membranes after the bacterial activity test: a) P1R against $S$. aureus, b) P1G against $S$. aureus and c) P3R against $E$. coli 
The results of drug release tests for the tested membrane are presented in Fig. 7. The ICP-MS method enabled us to determine the concentration of $\mathrm{SO}_{4}^{2-}$ ions, which corresponds directly to the concentration of gentamicin. In the case of ethacridine lactate, which is the colour active agent, concentration variability in the immersion medium was investigated by means of UV-Vis spectrophotometry.

Finally, a microbiological study was carried out to confirm the presence and release of gentamicin and ethacridine lactate (Fig. 8). The largest zone of inhibition of bacterial growth was observed for gentamicinmodified PLA fibres, especially against $E$. coli bacteria. The highest zone of inhibition was noticed for the porous P2G fibres $(21 \mathrm{~mm})$ and P3G $(23 \mathrm{~mm})$, compared to the non-porous ones (P1G, $18 \mathrm{~mm})$. The most effective was the membrane consisting of fibres with the largest pore diameter (P3G). Significantly lower efficiency was reported against Gram-positive bacteria for the membrane with gentamicin, again with higher responsiveness of membranes with porous fibres (P1G - $6 \mathrm{~mm}, \mathrm{P} 2 \mathrm{G}-8 \mathrm{~mm}, \mathrm{P} 3 \mathrm{G}-10 \mathrm{~mm}$ ). Membranes modified with ethacridine lactate showed the lack of inhibition zones around the membrane disk, for both Gram-negative and Gram-positive bacteria.

After testing the antibacterial activity, SEM pictures of membrane discs extracted from the agar substrate were also taken (Fig. 9). In the case of Grampositive bacteria, it can be clearly seen that the number of bacteria present on the P1 fibres with both ethacridine lactate (Fig. 9a) and gentamicin is comparable (Fig. 9b). On the other hand, much larger concentration of $E$. coli can be seen on the P3 fibres with ethacridine lactate (Fig. 9c).

\section{Discussion}

Current research shows that the use of modifications with active agents of an antibacterial nature is possible both at the stage preceding the production of porous and non-porous fibres and after their production, however, this is possible only when the appropriate active agents are selected. Gentamicin sulphate and ethacridine lactate used in this work are deliberately used here as their use provides comprehensive wound healing: from the elimination of bacterial biofilm (gentamicin) to inhibition of inflammation (ethacridine lactate).

As reported by Oie et al. [19], [20], in many cases impaired wound healing is the result of an underlying disease (e.g., autoimmunity, immunosuppression), and even stress. The use of a topical antiseptic drug, e.g., ethacridine lactate, is of clinical significance as it not only prevents additional weakening of the host's immune responses, but also positively support the immune system in its efforts to control wound infection. In turn, the use of gentamicin as a filler in the studied wound dressings can be supported by the fact that it is a parenterally-administered, broad-spectrum aminoglycoside antibiotic typically used for moderate to severe Gram-negative bacterial infections [2], [22], [25].

Due to the low solubility of gentamicin sulphate in organic solvents (DMSO $<1 \mathrm{mg} / \mathrm{ml}$, ethanol $<1 \mathrm{mg} / \mathrm{ml}$ ), it was used in the research in the form of a solid (powder) introduced into the spinning solution at a concentration of $5 \% \mathrm{wt} . \mathrm{v}$, exceeding the solubility reported in the literature $(5 \% \mathrm{wt} . / \mathrm{v}=52.6 \mathrm{mg} / \mathrm{ml})$ [2]. Thus, gentamicin was a specific fibre filler (Fig. 2), and the fact that gentamicin sulphate was used allowed for the use of sulphur as an analytical agent enabling identification of the active substance, e.g., in an immersion medium (Fig. 8). The standard ethacridine lactate solution used in clinical practice is $0.1 \%$ (water solubility $0.192 \mathrm{mg} / \mathrm{ml}$ ), which is enough to activate the immune system (including $\mathrm{T}$ leukocytes, granulocytes, monocytes). The choice of this modifier was justified by literature data, and so, for example, in the work of Oie and Kamiya, local antiseptic drugs such as ethacridine lactate deeply affected not only the inhibition of inflammation (secretion of antiinflammatory cytokines), but also activate the stage associated with wound healing by supporting the function of skin cells, such as: keratinocytes, fibroblasts [19], [12], [6].

Figures 3 and 4, which show the morphology of the fibres and their size distribution, clearly indicate a correlation between the solvent system applied and the morphology of the fibres. Careful selection of the solvent system and ambient conditions of the electrospinning process enabled the production of fibres with a non-porous (i.e., solid) and porous microstructure [10], [11], [18]. Porous fibres (P3) required solvents that are volatile and not miscible with water i.e., CHL and DCM, whose dielectric constant is low and the boiling point is similar. That made it possible to obtain a bimodal diameter distribution of the high porosity fibres. In turn, the combination of CHL and DMSO in the case of P2 fibres led to the unification of their diameters, while the use of these solvents for P1 fibres, but in a different ratio, can also lead to the production of porous fibres [14]. However, the CHL:DMSO combination provided the lowest average fibre size (max. $0.82 \mu \mathrm{m}$ for P2 fibres) using the same tip-collector distance (Fig. 3). It is also clear that 
the addition of gentamicin as a filler of PLA matrix increased the diameter of the fibres (Fig. 4).

The polymer stream extracted from the needle by a strong electric field is exposed to two synergistic effects: stretching of the polymer chain in the direction of the electric current and simultaneous evaporation of the solvent. During the jet ejection, the polymer solution constantly changes its composition, and the concentration of polymer in it increases due to solvents evaporation, which is mainly observed from the surface of the polymer jet. Comparing this phenomenon with the diffusion of solvent molecules from the core to the surface, the latter usually occurs more slowly [16], [23].

Luo et al. [21] showed that selection of solvents for electrospinning is a very important part of the experiment and they concluded that the solvent with the highest solubility is not necessarily the best choice for electrospinning. The balance of electrostatic forces between fluid particles is more important for obtaining smooth electrospun fibres, but solution conductivity, surface tension, and viscosity also matter. Wannatong et al. [30] confirmed that the dielectric constant is the most influential parameter in electrospinning solutions, important for charge distribution and increasing mass through put when exposed to an electric field. DMF is a poor solvent for PLA but has a high dipole moment and better conductivity than chloroform, as well as lower vapor pressure. When DMF was added in small amounts, it improved the electrospinnability of the solution, but at higher concentrations it affected PLA solubility [18].

Knowledge of the proportion and type of solvents is particularly important when fibres with a developed surface are obtained, i.e., for the production of porous fibres, the methods of vapor induced phase separation (VIPS) are used. The addition of a "weak" solvent or solution that does not dissolve the polymer but mixes well with water allows obtaining porosity in a humid environment using the VIPS method [18], [26]. Chloroform (CHL), which forms a binary solvent system of P2 membrane, evaporates faster than DMSO so the diffusion of remaining polymer molecules is limited. The use of DMSO as a polar solvent leads to the formation of more elliptical pores, and a porous skin forms on the surface of polymer jet after evaporation of the more volatile solvent, however, an increased amount of DMSO is observed in the fibre core [13]. The fibre core collapses under the influence of stretching forces, and by maintaining high humidity in the chamber, it is possible to create bulk porosity and high surface roughness. The binary solvent system of P3 membrane consists of non-polar solvents (DCM, CHL) with high volatility, and its faster evaporation creates a denser polymer jet. Also here, the solvents evaporate faster from the surface of polymer jet forming a porous skin, but, in this case, the core is stronger and neither collapses nor solidifies (Figs. 3 and 4). Electrospun polymeric fibres are usually characterized by lower crystallinity than the same polymer material obtained by another production technique: extrusion, slip casting [26].

The electrospinning process, which involves the vapor inducted phase separation (VIPS) and removal of solvents, has a significant impact on the glass transition temperature of polymer fibres, especially if solvents with different vapour pressures are used. Both modification processes: pre-electrospinning by addition of gentamicin to the PLA solution and the post-electrospinning modification (soaking of fibrous PLA membrane with ethacridine lactate) caused a shift in the glass transition temperature of the polymer $\left(T_{g}\right)$ by several degrees i.e., P1 $58.5^{\circ} \mathrm{C}$; P1G $62{ }^{\circ} \mathrm{C}$; P1R $65{ }^{\circ} \mathrm{C}$ (Table 2). In membranes consisting of porous fibres, the glass transition temperature increased after adding gentamicin as a filler (P2G, P3G) compared to fibrous membranes of neat polymer (P2, P3) and decreased for ethacridine lactate (P2R, P3R). The introduction of a solid filler, e.g., gentamicin, might lead to local ordering of the polymer structure (i.e., non porous materials $\mathrm{P} 1 \mathrm{G}$ ), however, the addition of gentamicin did not lead to an increase in the degree of polymer crystallinity, since the solvent system quickly evaporated as well as stopped the mobility of polymer chain, And the dispersed drug particles did not become nuclei in the fibres (i.e., porous materials $\mathrm{P} 2 \mathrm{G}$, P3G). In the case of the fibrous membrane soaked in ethacridine lactate solution, the drug is located on the surface of the fibres.

The effect of cold crystallization $\left(T_{c}\right)$ is observed in all materials, and changes in the maximum temperature of cold crystallization are clearly visible in membrane materials of type 3 (P3, P3G, P3R). The melting points $\left(T_{m}\right)$ of $\mathrm{P} 1 \mathrm{G}$ and $\mathrm{P} 1 \mathrm{R}$ are shifted from 159 to 169 and $161{ }^{\circ} \mathrm{C}$, respectively, and the same trend was followed by the membrane with bi-modal fibre distribution (P3). The peak of cold crystallization on DSC curves was observed in all porous and nonporous electrospun fibres, and indicated incomplete crystal formation during electrospinning (Table 2). These results confirmed the thesis about evaporation of the solvent prior to complete crystallization, which was also observed by Ribeiro et al. [23], [25].

As noted before, gentamicin is immiscible in any of the solvents used (CHL, DCM, DMF, low solubility in DMSO), nevertheless, there were no particles 
visible on the surface of the fibres, suggesting proper dispersion of the antibiotic within the fibres. For the same reason, due to the small amount of drug, the FTIR spectrum lacks characteristic bands in the range of $3600-3200 \mathrm{~cm}^{-1}$. The bands between $1000-1200 \mathrm{~cm}^{-1}$ correspond to the oscillatory vibrations of the $\mathrm{N}-\mathrm{H}$ and $\mathrm{C}-\mathrm{O}$ groups from gentamicin (Fig. 6a), with changes in absorbance intensity at $1056 \mathrm{~cm}^{-1}$ being particularly visible, and the absorption band at $895 \mathrm{~cm}^{-1}$ can be attributed to the $\mathrm{C}-\mathrm{O}-\mathrm{C}$ stretching vibration. A relatively small amount of the drug in the polymer fibres caused the lack of characteristic bands for gentamicin in the range of $3600-3200 \mathrm{~cm}^{-1}$. In turn, ethacridine lactate attached to the surface of the fibres is easily detected by the FTIR method, but its intensity is not high due to the low concentration of ethacridine lactate used. The bands associated with ethacridine lactate (the active substance of ethacridine lactate) are presented in Figs. $6 \mathrm{~b}$ and $6 \mathrm{c}$. The band observed at $1643 \mathrm{~cm}^{-1}$ can be attributed to the $\mathrm{C}=\mathrm{N}$ stretching vibration, and vibrations in the range of $3000-2800 \mathrm{~cm}^{-1}$ are associated with the $\mathrm{CH}_{2}-\mathrm{CH}_{3}$ group. The PLA fibres with ethacridine lactate also showed bands at $1578 \mathrm{~cm}^{-1}$ associated with asymmetric stretching vibration of lactate anion $\left(\mathrm{COO}^{-}\right)$and the aromatic stretching vibration in $\mathrm{C}=\mathrm{C}$ bands. The stretching band of the acridine ring is present at $1494 \mathrm{~cm}^{-1}$, and weak out-of-plane bending $\mathrm{C}-\mathrm{H}$ bands is present at 900 and $819 \mathrm{~cm}^{-1}$.

Table 2. Summary of characteristic temperatures measured by DSC

\begin{tabular}{|l|c|c|c|c|c|}
\hline Membrane & Material & $T_{g}\left[{ }^{\circ} \mathrm{C}\right]$ & $T_{c}\left[{ }^{\circ} \mathrm{C}\right]$ & $T_{m}\left[{ }^{\circ} \mathrm{C}\right]$ & $X_{c}[\%]$ \\
\hline \multirow{2}{*}{$\begin{array}{l}\text { with non-porous } \\
\text { fibres }\end{array}$} & $\mathrm{P} 1$ & 58.61 & 72.29 & 159.06 & 43.42 \\
\cline { 2 - 6 } & $\mathrm{P} 1 \mathrm{G}$ & 61.42 & 73.15 & 168.98 & 51.78 \\
\cline { 2 - 6 } & $\mathrm{P} 1 \mathrm{R}$ & 65.04 & 75.42 & 160.58 & 45.81 \\
\hline \multirow{2}{*}{$\begin{array}{l}\text { with porous } \\
\text { fibres }\end{array}$} & $\mathrm{P} 2$ & 57.46 & 73.60 & 159.07 & 47.49 \\
\cline { 2 - 6 } & $\mathrm{P} 2 \mathrm{G}$ & 62.25 & 76.34 & 160.98 & 47.33 \\
\cline { 2 - 6 } & $\mathrm{P} 2 \mathrm{R}$ & 50.14 & 75.25 & 157.00 & 37.34 \\
\hline \multirow{2}{*}{$\begin{array}{l}\text { with porous } \\
\text { fibres }\end{array}$} & $\mathrm{P} 3$ & 58.97 & 85.74 & 139.71 & 26.20 \\
\cline { 2 - 6 } & $\mathrm{P} 3 \mathrm{G}$ & 61.30 & 86.08 & 141.40 & 26.01 \\
\cline { 2 - 6 } & $\mathrm{P} 3 \mathrm{R}$ & 32.58 & 87.69 & 140.88 & 32.49 \\
\hline
\end{tabular}

Non-porous fibres (P1) can carry both gentamicin (P1G) and ethacridine lactate (P1R), and the lack of pores on the fibre structure does not discredit their ability to act in vivo as a drug carrier. The use of various types of modification methods resulted in differences in the amount of active agent released: gentamicin, which was introduced as a fibre filler, was released slowly (P1G), while for the same fibres with a smooth surface coated with ethacridine lactate, the effect was 300 times more intense. The more complex the fibre morphology (porous types P2, P3), the more effective the release. Regardless of whether the active compound was gentamicin or ethacridine lactate, fibres with monomodal distribution (on which $\mathrm{P} 2 \mathrm{G}$ and $\mathrm{P} 2 \mathrm{R}$ membranes were based) caused that much higher amount of active substance was carried to the environment than in the case of smoother fibres $(\mathrm{P} 1 \mathrm{G}$, P1R). Fibres with highly porous morphology (based on P3, Fig. 5) and bimodal fibres distribution were characterized by the fastest release of active agents. As can be seen from this analysis, the type of fibre and not the type of active substance or fibre modification method should be considered as the cause of the sustained trend for the release of gentamicin and ethacridine lactate.

In the tested fibres, the amount of gentamicin did not exceed $5 \% \mathrm{w} / \mathrm{w}$. Taking the recorded cumulative curves into account, it can be stated that gentamicin was released at the level of $20-30 \%$ from the porous fibres in the first $24 \mathrm{~h}$. It gives concentrations of $0.23-0.31 \mathrm{mg} / \mathrm{l}$ in the cases where the antibiotic carrier were $P 2 G$ and $P 3 G$ fibres, respectively. The maximum concentration of gentamicin released from the P2G fibre was observed after 4 days of incubation when about $60 \%$ of the drug was released (which gives a concentration of $0.63 \mathrm{mg} / \mathrm{l}$ ), further the drug concentration decreased. Slow release was observed in the P3G fibres, where the amount of released gentamicin reached about $50 \%$ (which gives the antibiotic concentration of $0.48 \mathrm{mg} / \mathrm{l}$ ). Even after 12 weeks of the incubation, the dose recommended by the Polish Pharmacopeia XI was not exceeded (the P3G fibres release about $0.82 \mathrm{mg} / \mathrm{l}$ ). Pores of about $116 \mathrm{~nm}$ diameter observed on the surface of the P2G fibres favoured faster release of the antibiotic, compared to the pores present in the $\mathrm{P} 3 \mathrm{G}$ fibres, which average diameter was $267 \mathrm{~nm}$. It was probably related to their depth - the VIPS technique in the solvent system used for the P2G material gives volumetric pores. While the solvent system used during electrospinning of the P3G fibres allowed to achieve surface porosity, which stopped the rapid ejection of gentamicin into the solution [14], [21]. The non-porous fibres kept low levels of gentamicin throughout the observation period.

The effectiveness of the drug-containing fibres was tested by UV-Vis and ICP-MS methods and microbiological test, the results of which are consistent and complement each other. The release of gentamicin (concentration $5 \mathrm{wt}$ \%) is lower in percentage (P1G) when the fibres have a non-porous surface (Figs. 7a-c). When the fibres have a porous structure on the surface, the release of drug is more intense and, consequently, increased bactericidal activity. The best results of the inhibition zone were obtained for the P3G 
membrane (Fig. 8), as a result of its microstructure. Large fibre diameters and larger pore size enabled greater drug incorporation into the fibres and easier drug release from the pores. It is well known that gentamicin is an effective bactericide against Gramnegative bacteria such as E. coli [15], [22], so it is not surprising that smaller zones of inhibition were have been observed for $S$. aureus. Ethacridine lactate is a substance that destroys harmful microorganisms or inhibits their activity, and this agent is classified as a drug for dermatological disorders for the treatment of wounds, ulcers, burns, scars, keloids or similar [20], [21]. It is mainly used at a concentration of $0.1 \%$ in an aqueous solution for surface application on the skin. The results of the microbiological activity test showed that there is no inhibition of bacterial growth outside the modified fibrous membrane (Figs. 9a-c). Selected bacteria strains are able to grow both aerobically and anaerobically, and the obtained fibrous structure of the membranes ensured free air flow, so the effect under the fibrous membrane is not the result of poor bacterial growth conditions, and the observed phenomena are probably only the bacteriostatic effect of the fibrous membrane soaked in ethacridine lactate solution [12].

\section{Conclusions}

The results of the investigation confirmed the successful volume (bulk) and surface modification of porous and solid (non-porous) fibres using drugs. Optimization of the conditions of the electrospinning process (mainly humidity), as well as the selection of appropriate solvents allowed to obtain porous fibres of various microstructures. It has been shown that depending on the fibre morphology and type of modification, different drug release efficiency can be achieved. Membranes with gentamicin showed bactericidal effect in contrast to the bacteriostatic nature of membranes with ethacridine lactate. To sum up, preliminary studies are a good starting point for further research on porous polylactide fibrous membranes for wound dressing.

\section{Acknowledgements}

This study was performed within the framework of funding for the statutory activities of AGH University of Science and Technology in Krakow, Faculty of Materials Science and Ceramics (11.11.160.182). The electrospinning / research/ was conducted using the equipment funded from Sonata 8 project granted by the
National Science Centre, No. 2014/15/D/ST5/02598. SEM imaging was supported by the infrastructure at International Centre of Electron Microscopy for Materials Science (IC-EM) at AGH University of Science and Technology.

\section{References}

[1] AmbeKar R.S., Kandasubramanian B., Advancements in nanofibers for wound dressing: A review, European Polymer Journal, 2019, 117, 304-336.

[2] Antibiotic Solubility Data Table TOKU-e, https://www.tokue.com/Antibiotic-Solubility-Data-Table.aspx

[3] Armentano I., Arciola C.R., Fortunati E., Ferrari D., Mattioli S., Rizzo J., The interaction of bacteria with engineered nanostructured polymeric materials: a review, The Scientific World Journal, 2014, 18.

[4] Aronson J.K. (ed.), Gentamicin. in: Meyler's side effects of drugs, (Sixteenth ed.), Elsevier, Oxford, 2016, 530-538.

[5] Changhua Chen, Yumin Chen, Pinpin Wu, Baoyuan Chen, Update on new medicinal applications of gentamicin: Evidence-based review, Journal of the Formosan Medical Association, 2014, (1 2), 72-82.

[6] Chouhan D., Dey N., Bhardwaj N., Mandal B.B., Emerging and innovative approaches for wound healing and skin regeneration: Current status and advances, Biomaterials, 2019, 216, 119-267.

[7] Dias J.R., Granja P.L., BÁRtolo P.J., Advances in electrospun skin substitutes, Progress in Materials Science, 2016, 1, 84, 314-334.

[8] Ethacridine lactate, PubChem https://pubchem.ncbi.nlm.nih.gov/ compound $/ 15789$

[9] Goertz O., Hirsch T., Ring A., Steinau H.U., Daigeler A., LEHNHARDT M., HomanN H.H., Influence of topically applied antimicrobial agents on muscular microcirculation, Ann. Plast. Surg., 2011, 67 (4), 407-412

[10] GRISTINA A.G., Biomaterial-centered infection: microbial adhesion versus tissue integration, Science, 1987, 25, 237 (4822), 1588-1595.

[11] Huang C., Thomas N.L., Fabricating porous poly(lactic acid) fibres via electrospinning, European Polymer Journal, 2018, 99, 464-476.

[12] Junka A., Bartoszewicz M., Smutnicka D., Secewicz A., SZYMCZYK P., Efficacy of antiseptics containing povidoneiodine, octenidine di-hydrochloride and ethacridine lactate against biofilm formed by Pseudomonas aeruginosaand Sta phylococcus aureus measured with the novel biofilm-oriented antiseptics test, Int. Wound J. 2014, 11, 730-734.

[13] Kotlarz M., Rainer J., Wegener E., Dobrzyński P., NeUnZehn J., Lederer A., Wolf-Brandstetter C., PAMUla E., SCHARNWEBER D., One step $3 D$ printing of surface functionalized composite scaffolds for tissue engineering applications, Acta Bioeng. Biomech., 2018, 20 (2), 35-45.

[14] Li Y., Lim C.T., KotaKi M., Study on structural and mechanical properties of porous PLA nanofibers electrospun by channelbased electrospinning system, Polymer., 2015, 56, 572-580.

[15] LuAn Y., Liu S., PiHL M., Mei H.C. van DER, LiU J., Hizal F., Bacterial interactions with nanostructured surfaces, Current Opinion in Colloid \& Interface Science. 2018, 38, 170-189.

[16] Luo C.J., NANGrejo M., Edirisinghe M., A novel method of selecting solvents for polymer electrospinning, Polymer, 2010, $51,1654-1662$. 
[17] Miguel S.P., Figueira D.R., Simões D., Ribeiro M.P., COUTINHO P., FERREIRA P., Electrospun polymeric nanofibres as wound dressings: A review, Colloids and Surfaces B: Biointerfaces, 2018, 169, 60-71.

[18] Qi Z., YU H., ChEN Y., ZHU M., Highly porous fibers prepared by electrospinning a ternary system of nonsolvent/ solvent/poly(l-lactic acid), Materials Letters. 2009, 63 (3), 415-418.

[19] OIE S., KamiYA A., Bacterial contamination of commercially available ethacridine lactate (acrinol) products, Journal of Hospital Infection, 1996, 1, 34 (1), 51-58.

[20] Oie S., KamiYa A., Particulate and microbial contamination in in-use admixed parenteral nutrition solutions, Biological and Pharmaceutical Bulletin, 2005, 28 (12), 2268-2270.

[21] PARK J.-Y., LEE I.-H., Controlled release of ketoprofen from electrospun porous polylactic acid (PLA) nanofibers, J. Polym. Res., 2011, 1, 18(6), 1287-1291.

[22] Phaechamud T., Issarayungyuen P., Pichayakorn W., Gentamicin sulfate-loaded porous natural rubber films for wound dressing, International Journal of Biological Macromolecules, 2016, 1, 85, 634-644.

[23] Ribeiro C., Sencadas V., Costa C.M., Ribelles J., LANCEROS-MÉNDEZ S., Tailoring the morphology and crystallinity of poly(Llactide acid) electrospun membranes, Sci. Technol. Adv. Mater, 2011, 12, 015001.

[24] Shi D., Mi G., Wang M., Webster T.J., In vitro and ex vivo systems at the forefront of infection modeling and drug discovery, Biomaterials, 2019, 198, 228-249.
[25] Simões D., Miguel S.P., Ribeiro M.P., Coutinho P., MENDOnÇA A.G., CORREIA I.J., Recent advances on antimicrobial wound dressing: A review, European Journal of Pharmaceutics and Biopharmaceutics, 2018, 127, 130-141.

[26] Smyth M., Poursorkhabi V., Mohanty A.K., Gregori S., MisRA M., Electrospinning highly oriented and crystalline poly(lactic acid) fiber mats, J. Mater. Sci., 2014, 49, 2430-2441.

[27] Stodolak-Zych E., Dzierzkowska E., Matwally S., MikOlAJCZYK M., GAJEK M., RAPACZ-KMitA A., Multifunctional porous membranes with antibacterial properties, International Journal of Polymeric Materials and Polymeric Biomaterials, 2019, 11, 68 (1-3), 19-26.

[28] Stodolak-Zych E., Rozmus K., DZIERZKowska E., ZyCh Ł., RapaCZ-KMita A., Gargas M., KoŁaczKowsKa E., CiEniawsKa M., KsiĄŻEK K., ŚCISŁOWSKA-CZARnECKA A., The membrane with polylactide and hyaluronic fibers for skin substitute, Acta Bioeng. Biomech., 2018, Vol. 20, No. 4, 91-99.

[29] Turek A., Kasperczyk J., Jelonek K., Borecka A., JANECZeK H., Libera M., GrUChliK A., DobrZyŃSKi P., Thermal properties and morphology changes in degradation process of poly(L-lactide-co-glycolide) matrices with risperidone, Acta Bioeng. Biomech., 2015, 17 (1), 11-20.

[30] Wannatong L., Sirivat A., Supaphol P., Effects of solvents on electrospun polymeric fibers: preliminary study on polystyrene, Polym. Int., 2004, 53, 1851-1859. 